\title{
Eine vergleichende Analyse der Basisfallwerte in der Schweiz und in Deutschland
}

\author{
In Deutschland gibt es bundesländerspezifische Basisfallwerte, in der Schweiz exis- \\ tieren keine einheitlichen Baserates. Bei einem Vergleich der Basisfallwerte beider \\ Länder müssen diverse Unterschiede berücksichtigt werden. Dann zeigt sich, dass \\ die Werte enger beieinander liegen, als es zunächst scheint.
}

\author{
Robert Kopfa, \\ Agnes Genewein ${ }^{b}$, \\ Lukas Erbc, \\ Conrad E. Müller ${ }^{d}$ \\ a Dipl. Betriebswirt(FH), \\ Controller \\ b Dr. med., Oberärztin \\ c lic. rer. pol., $\mathrm{CFO}$ \\ d Dr. med., CEO
}

* Die Literaturangaben finden sich unter www.saez.ch $\rightarrow$ aktuelle Nummer oder $\rightarrow$ Archiv $\rightarrow 2014 \rightarrow 44$.

Korrespondenz: Robert Kopf Universitäts-Kinderspital beider Basel (UKBB)

Spitalstrasse 33

CH-4031 Basel

Tel. 0617041735

robert.kopf[at]ukbb.ch

\section{Einleitung}

Sowohl in der Schweiz als auch in Deutschland werden stationäre Krankenhausleistungen nach Fallpauschalen abgerechnet. Das Schweizer System (SwissDRG) basiert auf dem System German-DRG (G-DRG) [1]*. Gleichzeitig jedoch wurde es an die Besonderheiten des Gesundheitssystems der Schweiz angepasst. GDRG ist die deutsche Adaptation an das australische DRG-Systems (AR-DRG). Die Entwicklungszusammenarbeit mit dem deutschen Institut für das Entgeltsystem im Krankenhaus (InEK) lief auf Ende 2012 aus. Die SwissDRG AG entwickelt das SwissDRG-System ab diesem Zeitpunkt selbständig weiter [1].

Hier bietet sich ein Vergleich der Basisfallwerte beider Länder geradezu an. So stehen in der Schweiz Universitätsspitälern mit einer Baserate von rund 11000 Franken fast das 3-Fache von Krankenhäusern in Baden-Württemberg zur Verfügung. Eine nähere Betrachtung ist jedoch zwingend erforderlich. Hierbei sind folgende Einflussfaktoren näher zu analysieren: Finanzierung der Investitionskosten, Unterschiede bei Kaufkraft und DRG-Kataloge sowie Vergütung von Zusatzentgelten.

Diese Effekte wurden am Beispiel des UniversitätsKinderspitals beider Basel (UKBB) quantifiziert, lassen sich aber mit Einschränkungen auch auf die übrigen (Universitäts-)Spitäler in der Schweiz übertragen.

Der Artikel widmet sich der Analyse dieser Vergleichbarkeit der auf den ersten Blick doch deutlichen Unterschiede der Basisfallwerte.

\section{Unterschiedliche Basisfallwerte}

In der Schweiz gibt es im Gegensatz zu Deutschland keine einheitliche Baserate. Die Spitäler verhandeln getrennt, jedoch kristallisieren sich gleichgelagerte Tendenzen bei den Basisfallwerten heraus. Dagegen wurde in Deutschland nach Beendigung der Konvergenzphase (Beginn 2009) ein für die Bundesländer einheitlicher Basisfallwert etabliert. Dabei spielt Grösse oder Versorgungsstufe des Hauses keine Rolle. Dieser liegt für Baden-Württemberg inkl. Ausgleichen bei 3121.04 Euro. Dies sind umgerechnet 3841 CHF [2]. Die Bandbreite im Jahr 2013 liegt zwischen 2999.85 Euro für Thüringen und 3250.70 Euro für RheinlandPfalz.

\section{Analyse comparative des prix de}

\section{base DRG en Suisse et en Allemagne}

Depuis le $1^{\text {er }}$ janvier 2012, les hôpitaux facturent leurs prestations stationnaires au moyen des DRG (forfaits par cas). Le système SwissDRG se fonde sur le système German-DRG qui a été adapté aux spécificités du système de santé suisse. La convention de coopération entre SwissDRG SA et I'Institut allemand InEK, spécialisé dans la rémunération des coûts hospitaliers, est arrivée à échéance à fin 2012. Depuis la fin de la phase de convergence, il existe en Allemagne des prix de base spécifiques à chaque Etat fédéré. En Suisse en revanche, il n'existe pas de prix de base uniforme. Les hôpitaux négocient séparément les tarifs, même si des tendances similaires se dessinent au niveau des prix de base. L'article ci-après montre quelles divergences doivent être prises en compte lors de la comparaison de ces différents prix de base. Les prix de base sont plus proches en Allemagne et en Suisse que leur valeur nominale pourrait le laisser croire à première vue. La comparaison effectuée permet d'aboutir à la conclusion que les différences du prix de base entre les deux systèmes présentent une explication plausible et qu'elles sont quasiment nulles après élimination des divergences.

Für die Unispitäler Basel, Bern und Zürich liegt der Durchschnittswert für 2013 bei einem Wert von 11022 CHF. Der Preisüberwacher [3] sieht für die Universitätsspitäler einen Wert von 9674 CHF (100\%, inkl. Anlagenutzungskosten) vor. Für Nicht-Universitätsspitäler einen Wert von 8974 CHF. 


\section{DRG-Systematik}

In Deutschland wurde zur Abrechnung von Akutkrankenhausleistungen 2003 optional und 2004 verbindlich auf Fallpauschalen umgestellt. Pate bei der Entwicklung der Systematik war das australische DRGSystem. Ausgenommen hiervon sind grundsätzlich Abteilungen und Spitäler für Psychiatrie, Psychosomatik und psychotherapeutische Medizin. Das DRGSystem ist nach § 17b KHG (Krankenhausfinanzierungsgesetz) ständig weiterzuentwickeln. Diese Aufgabe liegt in den Händen des InEK [4] (Institut für das Entgeltsystem im Krankenhaus).

«Für Leistungen, die noch nicht mit den DRG-Fallpauschalen und Zusatzentgelten sachgerecht vergütet werden können und für besondere Einrichtungen ...» können die Einrichtungen mit den Krankenkassen fall- oder tagesbezogene Entgelte oder in Ausnahmefällen auch Zusatzentgelte vereinbaren [5]. Die Ausführungen in § 17b Absatz 1 Satz 15 KHG (Krankenhausfinanzierungsgesetz) konkretisiert dies auf die besonderen medizinischen Leistungen, die Häufung schwerkranker Patienten oder auf Gründe im Rahmen der Versorgungsstruktur.

\section{G-DRG System 2013}

Eckwerte zum G-DRG-System in der Systematik 2013: 1187 DRGs, davon sind 1142 bewertet und 40 nicht bewertet (Anlage 3a). Im Zusatzentgelte-Katalog (Anlage 2 FPV 2013) werden 90 bewertete Zusatzentgelte aufgeführt. Die Anzahl der krankenhausindividuell zu vereinbarenden Zusatzentgelte gem. §6 Abs. 1 KHEntgG (Anlage 4 FPV 2013) liegt bei 66.

\section{SwissDRG}

Das System SwissDRG 2.0 für 2013 enthält 991 DRGs davon sind 973 mit Kostengewichten bewertet. Es sind 5 Zusatzentgelte definiert - 3 davon sind bewertet.

Ein grosser Unterschied beider Systeme liegt im Bereich der Zusatzentgelte. «Zusatzentgelte werden für die Vergütung von teuren Medikamenten, Blutprodukten, Implantaten und kostenintensiven Behandlungsmethoden eingesetzt, die über mehrere Fallgruppen streuen.» [6] Hier stehen 2013 im System SwissDRG 5 Zusatzentgelten 156 im G-DRG gegenüber.

\section{DRG-Systemvergleich \\ Einleitung}

Ein Vergleich der beiden DRG-Kataloge SwissDRG versus G-DRG soll zeigen, welche Unterschiede in der Bewertung der einzelnen Fallpauschalen vorhanden sind. Die nachfolgenden Analysen beinhalten die IstDaten bezüglich Fallzahlen, Verweildauern und Case Mix effektiv des UKBB aus dem Jahr 2013.

Im Universitäts-Kinderspital beider Basel wurden im Jahr 2013 insgesamt 393 unterschiedliche DRGs abgerechnet. Damit belegt das Kinderspital 40,5\% der 973 bewerteten DRGs

\section{Vorgehensweise und Annahmen}

Weitere Prämissen sind jedoch zu beachten. Bei der
Gegenüberstellung der einzelnen DRGs basiert die Zuordnung auf dem 4-stelligen DRG-Code. Basis ist das SwissDRG-System 2.0. Beim Fehlen einer entsprechenden Fallpauschale im G-DRG-System wurde das Kostengewicht des Schweizer DRG-Systems stellvertretend eingesetzt. Dies war bei insgesamt 123 DRGs der 973 DRGs der Fall, obwohl der Katalog G-DRGs mit insgesamt 1142 DRGs um 169 Fallpauschalen grösser ist als SwissDRG.

Ein tiefergehender Vergleich ist leider nicht möglich, da unterschiedliche Systematiken der OPS-Codes zur Leistungskodierung eingesetzt werden. So wird im Entgeltsystem G-DRG der OPS eingesetzt. Der OPS ist eine Adaption der Internationalen Klassifikation der Prozeduren in der Medizin (ICPM) der Weltgesundheitsorganisation (WHO) [7]. Der deutsche Prozedurenkatalog OPS enthält weit über 20000 Einträge und ist damit um rund das Doppelte umfangreicher als der schweizerische Prozedurenkatalog CHOP.

\section{Vergleich Case Mix der beiden Kataloge}

Vergleich DRGs nach Katalog Kostengewichte nach effektivem Kostengewicht

Unter Verwendung der Abrechnungsdaten des UKBB und den o.g. Prämissen zur Vergleichbarkeit beider DRG-Kataloge zeigt Tabelle 1. Hierbei wurden die Fallzahlen 2013 mit dem jeweiligen Kostengewicht der entsprechenden DRG multipliziert. Unter Berücksichtigung der jeweiligen $\mathrm{Zu}$ - und Abschläge zeigt Tabelle 2 das Ergebnis. Alles in allem weisen die beiden Systeme in der Gesamtsicht eine Differenz von 27,3\% bzw. 30,7\% aus.

\section{Besonderheiten des DRG-Vergleichs}

Auffällig ist die MDC 15: Bei Neugeborenen liegt das effektive Kostengewicht im G-DRG um 66,7\% höher als im SwissDRG-System. Dies wird hauptsächlich durch folgende 2 Faktoren verursacht: Die Vergütung für Neugeborene < $1000 \mathrm{~g}$. Im G-DRG-System existieren hierfür 5 weitere Gruppen. Im SwissDRG dagegen werden diese Patienten in einer Gruppe Neugeborene $<1000 \mathrm{~g}$ zusammengefasst. D.h., ein Frühgeborenes $<600 \mathrm{~g}$ erhält im G-DRG-System mit der Fallpauschale P61A (Neugeborenes, Aufnahmegewicht $<600 \mathrm{~g}$ mit signifikanter OR-Prozedur) ein Kataloggewicht von 43.3 Punkten - im SwissDRG fällt es in die Gruppe <1000 g mit einem Kostengewicht von 21.45 Punkten. Gleiches gilt für Neugeborene $<600$ g ohne signifikante OR-Prozedur.

Eine weitere auffällige Differenz bei einer durchaus signifikanten Fallzahl liegt bei der DRG P05C (Neugeborenes, Aufnahmegewicht $>1999$ g mit signifikanter OR-Prozedur oder Beatmung > 95 Stunden, ohne mehrere schwere Probleme) vor. Ist im G-DRG hierfür ein Kataloggewicht von 5.8 Punkten vorgesehen, stehen im SwissDRG lediglich 2.6 Case Mix-Punkte dagegen.

Ersetzt man in diesen beiden DRG-Gruppen die in Deutschland vergüteten Werte, sinkt die Differenz beider Kataloge in der MDC 15 von 61\% auf $11 \%$. 
Tabelle 1

Vergleich Case Mix nach Katalog.

Major Diagnostic Category (MDC)

\begin{tabular}{|c|c|c|}
\hline $\begin{array}{r}\text { Summe von } \\
\text { UKBB SwissDRG } \\
\text { Katalog CW }\end{array}$ & $\begin{array}{r}\text { Summe von } \\
\text { UKBB G-DRG } \\
\text { Katalog CW }\end{array}$ & $\begin{array}{r}\text { Summe von } \\
\text { Abweichung }\end{array}$ \\
\hline 366,815 & 462,343 & $26,0 \%$ \\
\hline 509,922 & 491,114 & $-3,7 \%$ \\
\hline 19,333 & 17,720 & $-8,3 \%$ \\
\hline 380,121 & 423,328 & $11,4 \%$ \\
\hline 471,900 & 588,853 & $24,8 \%$ \\
\hline 26,961 & 26,754 & $-0,8 \%$ \\
\hline 427,994 & 451,795 & $5,6 \%$ \\
\hline 12,455 & 11,970 & $-3,9 \%$ \\
\hline 1347,156 & 1874,756 & $39,2 \%$ \\
\hline 234,457 & 242,769 & $3,5 \%$ \\
\hline 98,464 & 100,073 & $1,6 \%$ \\
\hline 119,556 & 143,277 & $19,8 \%$ \\
\hline 65,432 & 99,231 & $51,7 \%$ \\
\hline 18,464 & 17,330 & $-6,1 \%$ \\
\hline - & - & \\
\hline 1374,979 & 2214,107 & $61,0 \%$ \\
\hline 49,821 & 62,638 & $25,7 \%$ \\
\hline 223,630 & 272,132 & $21,7 \%$ \\
\hline - & - & \\
\hline 101,818 & 102,196 & $0,4 \%$ \\
\hline 262,193 & 258,686 & $-1,3 \%$ \\
\hline 7,142 & 6,995 & $-2,1 \%$ \\
\hline 13,637 & 17,068 & $25,2 \%$ \\
\hline 81,059 & 68,569 & $-15,4 \%$ \\
\hline 52,336 & 35,456 & $-32,3 \%$ \\
\hline 40,616 & 37,879 & $-6,7 \%$ \\
\hline 17,457 & 24,113 & $38,1 \%$ \\
\hline 6323,718 & 8051,152 & $27,3 \%$ \\
\hline
\end{tabular}

\section{00 Prä-MDC}

MDC 01 Krankheiten und Störungen des Nervensystems

MDC 02 Krankheiten und Störungen des Auges

MDC 03 Krankheiten und Störungen des Ohres, der Nase, des Mundes und des Halses

MDC 04 Krankheiten und Störungen der Atmungsorgane

MDC 05 Krankheiten und Störungen des Kreislaufsystems

MDC 06 Krankheiten und Störungen der Verdauungsorgane

MDC 07 Krankheiten und Störungen an hepatobiliärem System und Pankreas

MDC 08 Krankheiten und Störungen an Muskel-Skelett-System und Bindegewebe

MDC 09 Krankheiten und Störungen an Haut, Unterhaut und Mamma

MDC 10 Endokrine, Ernährungs- und Stoffwechselkrankheiten

MDC 11 Krankheiten und Störungen der Harnorgane

MDC 12 Krankheiten und Störungen der männlichen Geschlechtsorgane

MDC 13 Krankheiten und Störungen der weiblichen Geschlechtsorgane

MDC 14 Schwangerschaft, Geburt und Wochenbett

MDC 15 Neugeborene

MDC 16 Krankheiten des Blutes, der blutbildenden Organe und des Immunsystems

MDC 17 Hämatologische und solide Neubildungen

MDC 18 A HIV

MDC 18B Infektiöse und parasitäre Krankheiten

MDC 19 Psychische Krankheiten und Störungen

8051,152

\section{Effekt Zusatzentgelte}

\section{Grundlagen}

Das System SwissDRG beinhaltet für 2013 insgesamt 5 Zusatzentgelte. Dem stehen im G-DRG-System 156 ZEs gegenüber. Bei Zusatzentgelten wird zwischen bewerteten Entgelten (siehe Anlage 2/5 der Fallpauschalenvereinbarung) sowie krankenhausindividuell $\mathrm{zu}$ vereinbarenden Entgelten (siehe Anlage 4/6 der Fallpauschalenvereinbarung) unterschieden.

\section{Welchen Anteil haben die Zusatzentgelte am DRG-Erlösvolumen?}

Dieser Wert lag für deutsche Krankenhäuser für die Jahre 2005 bis 2007 pro Bettenklasse vor und zeigt einen stetig wachsenden Wert. Für Krankenhäuser mit bis zu 50 Betten betrug der Anteil 2007 1,1\% und bei Häusern mit über 1000 Betten 4,5\% (2005: 3,5\%) [8].

Für alle Spitäler in Baden-Württemberg wird im Krankenhaus-Report 2014 [9] ein Budgetanteil Zusatzentgelte für 2012 in Höhe von 3,3\% ausgewiesen.
Im Krankenhaus-Report 2013 ist für alle Krankenhäuser Deutschlands ein Anteil für Zusatzentgelte 2010 von 2,6\% sowie für 2011 von 2,8\% ausgewiesen. Zusätzlich wird im Geschäftsbericht der BWKG (BadenWürttembergische Krankenhausgesellschaft) von einer positiven Ausweitung des Zusatzentgeltkatalogs gesprochen. Mit zunehmender Güte des G-DRG-Systems erfolgt eine stärkere Identifikation zusatzentgeltfähiger Leistungen. 143 Entgelten 2010 standen $83 \mathrm{im}$ Jahr 2006 gegenüber [10].

Hieraus folgt, dass seit Einführung des G-DRGSystems die Anzahl von abrechenbaren Zusatzentgelten sowie deren Budgetanteil kontinuierlich stieg. Ebenso zeigt sich, dass der Budgetanteil von ZEs mit der Spitalgrösse korreliert. Dies ist insbesondere auf teure Medikamente, welche zum grossen Teil in der Onkologie zum Einsatz kommen, zurückzuführen. Da das UKBB eine eigene onkologische Station betreibt sowie Stammzelltransplantation durchführt, kann es einem Spital mit einer Bettenzahl >1000 gleichgestellt werden. Unter Berücksichtigung dieser 
Tabelle 2

Vergleich Case Mix effektiv.

Major Diagnostic Category (MDC)

\begin{tabular}{|c|c|c|}
\hline $\begin{array}{r}\text { Summe von } \\
\text { UKBB SwissDRG } \\
\text { Effektiv CW }\end{array}$ & $\begin{array}{r}\text { Summe von } \\
\text { UKBB G-DRG } \\
\text { Effektiv CW }\end{array}$ & $\begin{array}{l}\text { Abweichung } \\
\text { Effektiv CW }\end{array}$ \\
\hline 370,315 & 469,325 & $26,7 \%$ \\
\hline 422,902 & 429,194 & $1,5 \%$ \\
\hline 16,388 & 15,067 & $-8,1 \%$ \\
\hline 346,293 & 376,961 & $8,9 \%$ \\
\hline 401,862 & 465,946 & $15,9 \%$ \\
\hline 19,550 & 18,643 & $-4,6 \%$ \\
\hline 389,644 & 408,908 & $4,9 \%$ \\
\hline 12,570 & 12,760 & $1,5 \%$ \\
\hline 1294,895 & 1781,296 & $37,6 \%$ \\
\hline 167,904 & 175,651 & $4,6 \%$ \\
\hline 95,444 & 98,933 & $3,7 \%$ \\
\hline 110,952 & 132,104 & $19,1 \%$ \\
\hline 62,755 & 90,373 & $44,0 \%$ \\
\hline 17,835 & 16,476 & $-7,6 \%$ \\
\hline - & - & \\
\hline 1626,111 & 2710,143 & $66,7 \%$ \\
\hline 46,327 & 57,126 & $23,3 \%$ \\
\hline 230,070 & 274,279 & $19,2 \%$ \\
\hline - & - & \\
\hline 90,495 & 90,391 & $-0,1 \%$ \\
\hline 339,723 & 362,602 & $6,7 \%$ \\
\hline 3,577 & 3,482 & $-2,7 \%$ \\
\hline 14,256 & 17,196 & $20,6 \%$ \\
\hline 55,025 & 48,559 & $-11,8 \%$ \\
\hline 50,800 & 35,550 & $-30,0 \%$ \\
\hline 28,308 & 30,192 & $6,7 \%$ \\
\hline 18,361 & 23,769 & $29,5 \%$ \\
\hline 6232,360 & 8144,926 & $30,7 \%$ \\
\hline
\end{tabular}

\section{00 Prä-MDC}

MDC 01 Krankheiten und Störungen des Nervensystems

MDC 02 Krankheiten und Störungen des Auges

MDC 03 Krankheiten und Störungen des Ohres, der Nase, des Mundes und des Halses

MDC 04 Krankheiten und Störungen der Atmungsorgane

MDC 05 Krankheiten und Störungen des Kreislaufsystems

MDC 06 Krankheiten und Störungen der Verdauungsorgane

MDC 07 Krankheiten und Störungen an hepatobiliärem System und Pankreas

MDC 08 Krankheiten und Störungen an Muskel-Skelett-System und Bindegewebe

MDC 09 Krankheiten und Störungen an Haut, Unterhaut und Mamma

MDC 10 Endokrine, Ernährungs- und Stoffwechselkrankheiten

MDC 11 Krankheiten und Störungen der Harnorgane

MDC 12 Krankheiten und Störungen der männlichen Geschlechtsorgane

MDC 13 Krankheiten und Störungen der weiblichen Geschlechtsorgane

MDC 14 Schwangerschaft, Geburt und Wochenbett

MDC 15 Neugeborene

MDC 16 Krankheiten des Blutes, der blutbildenden Organe und des Immunsystems

MDC 17 Hämatologische und solide Neubildungen

MDC 18 A HIV

MDC 18B Infektiöse und parasitäre Krankheiten

MDC 19 Psychische Krankheiten und Störungen

8144,926

Entwicklung kann für das UKBB ein Budgetanteil 2013 für ZEs von mind. 6\% im Umfeld G-DRG angenommen werden.

\section{Preiseffekt}

Ein Blick auf das Preisvergleichsportal [11] zeigt: Identische Produkte sind in der Schweiz massiv teurer als beispielsweise in Deutschland. Um dies auszugleichen, ist dementsprechend natürlich auch das Lohn- und Gehaltsniveau in der Schweiz höher. Im Endeffekt wirkt dies sich dann auch auf die Baserate für die Spitäler aus.

Ein einfacher Benchmark ist in diesem Zusammenhang der Big-Mac-Index. Er vergleicht die Preise des Big Macs in verschiedenen Ländern der Erde. Durch die Umrechnung der inländischen Währungen zum USDollar-Kurs werden diese untereinander verglichen. Der Big Mac bietet einen einfachen Indikator für das Preisniveau einer Volkswirtschaft. Es gibt ihn fast überall auf der Welt in standardisierter Grösse, Zusammensetzung und Qualität. Siehe hierzu Abbildung 1.
Der Preisunterschied zwischen der Schweiz und Deutschland betrug in diesem Index für Januar 2014 43,3\%. Bei Berücksichtigung von Wechselkursveränderungen rund 42\% für das Gesamtjahr 2013.

Das Bundesamt für Statistik führt aus: «Ein bestimmter Warenkorb mit identischem Nutzen (einem Teil des Bruttoinlandprodukts) kostete im Jahr 2012 in der Schweiz 185 CHF, in Deutschland 103 EUR, in Frankreich 112 EUR, in Italien 100 EUR und in Österreich 110 EUR. Im Durchschnitt der 28 EU-Mitgliedsländer kostete er 100 EUR» [13]. Unter Annahme eines Wechselkurses von 1,23 ergeben sich Mehrkosten in der Schweiz gegenüber Deutschland von $46 \%$.

\section{Lohnniveau}

Bestätigt werden die Aussagen zum Preisniveau durch einen Benchmark der Lohnkosten für den Pflegedienst. Nach der Krankenhausstatistik Baden-Württemberg [14] aus dem Jahre 2012 betragen die durchschnittlichen Lohnkosten für den Pflege- und Funk- 
tionsdienst 67506 CHF. Bei einer Tarifsteigerung für 2013 von rund 3\% beträgt der Wert für 201369531 CHF und liegt damit um über $40 \%$ unter dem Niveau vom UKBB.

\section{Investitionseffekt}

Mit der Revision KVG ist im Schweizer Gesundheitswesen auch die Investitionsfinanzierung in der Fallpauschalenvergütung enthalten.

Der Investitionskostenzuschlag (IKZ) regelt den Abgeltungsmechanismus für die Erstellung und Nutzung der betrieblichen (mobilen und immobilen) Sachanlagen. Für das Jahr 2012 hat der Bund den IKZ auf $10 \%$ festgesetzt. Für das Jahr 2013 sollten ihn die Vertragspartner mangels bundesrätlichen Entscheids selber festlegen oder direkt einrechnen.

In Deutschland teilen sich seit dem Inkrafttreten des Krankenhausfinanzierungsgesetzes von 1972 die Bundesländer und die gesetzlichen Krankenkassen die Krankenhausfinanzierung: Die Investitionskosten werden im Wege der öffentlichen Förderung durch die Bundesländer getragen, die Krankenkassen finanzieren die laufenden Betriebskosten im stationären Bereich grösstenteils über die Fallpauschalen. Damit sind die Investitionskosten in Deutschland nicht in den Basispreisen enthalten.

\section{Fazit und Variantenbildung}

Unter Berücksichtigung der o.g. Faktoren zeigt sich, dass die Basisfallwerte in der Schweiz und in Deutschland enger beieinander liegen, als ihr nomineller Wert es auf den ersten Blick erscheinen lässt. Tabelle 3 zeigt, dass unter Berücksichtigung der genannten Faktoren in einem Ländervergleich die DRG-FallpauschalenVergütung in der Schweiz im Mittel nur leicht (11\%) über der Vergütung in Baden-Württemberg liegt, trotz der auf den ersten Blick deutlich höheren nominellen Baserate im Schweizer DRG-System.

\section{Abbildung 1}

Big-Mac-Index: Preis für einen Big Mac in ausgewählten Ländern im Januar 2014.

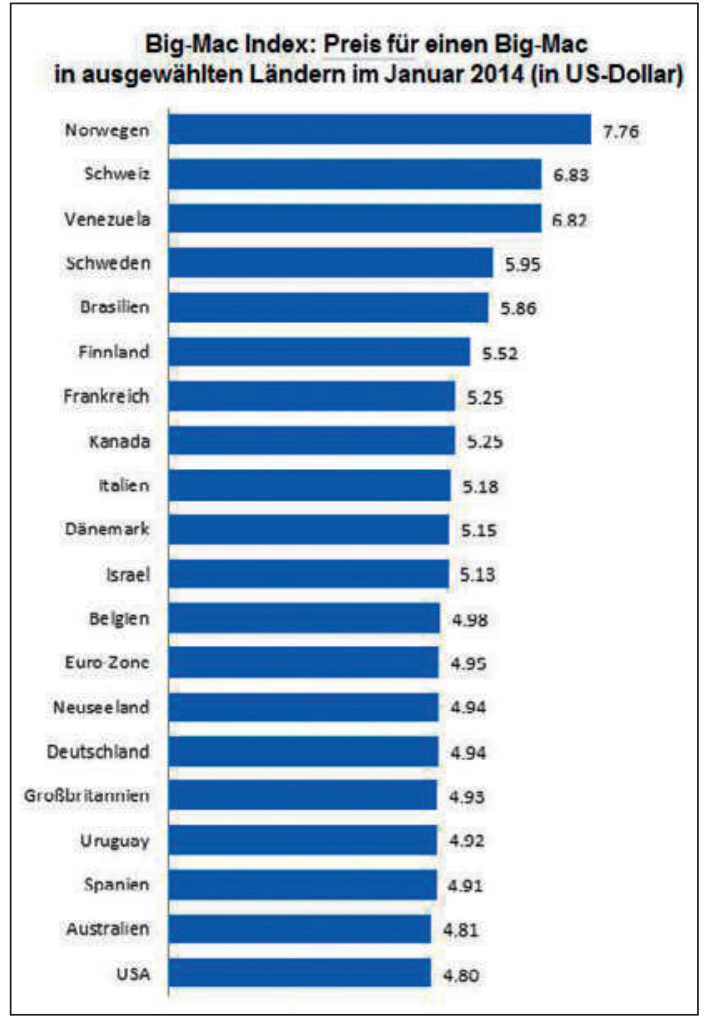

Die Analyse zeigt auf, dass ein einfacher Vergleich der in den jeweiligen Ländern zur Abrechnung kommenden Baserate sachlich nicht gerechtfertigt ist. Es kann vielmehr festgehalten werden, dass die Unterschiede in den Basisfallwerten zwischen beiden Systemen plausibel erklärt werden können und die Differenz nach Eliminierung der systemimmanenten Faktoren gegen Null tendiert.

Tabelle 3

Vergleich Basisfallwerte Deutschland/Schweiz mit unterschiedlichen Varianten.

\begin{tabular}{|c|c|c|c|c|c|c|c|c|c|}
\hline \multirow{2}{*}{$\begin{array}{l}\text { Beschreibung } \\
\text { Basisfallwert } 2013\end{array}$} & \multicolumn{3}{|c|}{ Variante I } & \multicolumn{3}{|c|}{ Variante II } & \multicolumn{3}{|c|}{ Variante III } \\
\hline & Faktor & \multicolumn{2}{|c|}{ Ergebnis } & Faktor & \multicolumn{2}{|c|}{ Ergebnis } & Faktor & \multicolumn{2}{|c|}{ Ergebnis } \\
\hline \multirow[t]{2}{*}{ Baden Württemberg } & & 3121 & Euro & & 3121 & Euro & & 3121 & Euro \\
\hline & & 3841 & $\mathrm{CHF}$ & & 3841 & $\mathrm{CHF}$ & & 3841 & $\mathrm{CHF}$ \\
\hline Ø Basisfallwert Schweiz 2013 & & 11000 & $\mathrm{CHF}$ & & 11000 & $\mathrm{CHF}$ & & 11000 & $\mathrm{CHF}$ \\
\hline Investitionsanteil & $-10,0 \%$ & -1100 & $\mathrm{CHF}$ & $-10,0 \%$ & -1100 & $\mathrm{CHF}$ & $-10,0 \%$ & -1100 & $\mathrm{CHF}$ \\
\hline Basisfallwert vergleichbar I & & 9900 & $\mathrm{CHF}$ & & 9900 & $\mathrm{CHF}$ & & 9900 & $\mathrm{CHF}$ \\
\hline CH Preiseffekt & $-42,0 \%$ & -4158 & $\mathrm{CHF}$ & $-38,5 \%$ & -3812 & $\mathrm{CHF}$ & $-35,0 \%$ & -3465 & $\mathrm{CHF}$ \\
\hline Zwischensumme & & 5742 & $\mathrm{CHF}$ & & 6089 & $\mathrm{CHF}$ & & 6435 & $\mathrm{CHF}$ \\
\hline DRG-Effekt & $-30,0 \%$ & -1723 & $\mathrm{CHF}$ & $-25,0 \%$ & -1522 & $\mathrm{CHF}$ & $-20,0 \%$ & -1287 & $\mathrm{CHF}$ \\
\hline Zusatzentgelt-Effekt & $-6,0 \%$ & -345 & $\mathrm{CHF}$ & $-5,0 \%$ & -304 & $\mathrm{CHF}$ & $-4,0 \%$ & -257 & $\mathrm{CHF}$ \\
\hline Basisfallwert vergleichbar II & & 3675 & $\mathrm{CHF}$ & & 4262 & $\mathrm{CHF}$ & & 4891 & $\mathrm{CHF}$ \\
\hline Differenz & & -166 & CHF & & 421 & $\mathrm{CHF}$ & & 1049 & CHF \\
\hline Verhältnis & & 0,96 & & & 1,11 & & & 1,27 & \\
\hline
\end{tabular}




\section{Literatur}

1 www.fischer-zim.ch/text-pcssa-pdf/t-ga-H9-SystemSwiss-DRG-0003.pdf, S. 3

2 www.estv.admin.ch/wehrpflichtersatzabgabe/ dienstleistungen/00263/?lang=de

3 www.preisueberwacher.admin.ch

4 www.g-drg.de/cms/

5 §6 Vereinbarung sonstiger Entgelte KHEntgG

6 www.drgs.ch/zusatzentgelte.html

7 www.dimdi.de

8 Klauber J, Robra B-P, Schellschmidt H (Hrsg.). Krankenhaus-Report 2008/2009: S. 368.
9 Klauber J, Geraedts M, Friedrich J, Wasem J (Hrsg.). Krankenhaus-Report 2014: S. 269.

10 www.bwkg.de/die-bwkg/geschaeftsbericht.html für 2009 bis 2011, S. 17.

11 www.preisbarometer.ch

12 http://de.statista.com/statistik/daten/studie/199335/ umfrage/big-mac-index--weltweiter-preis-fuer-einenbig-mac/

13 www.bfs.admin.ch/bfs/portal/de/index/ themen/05/07/blank/key/02.html

14 www.statistik-bw.de 